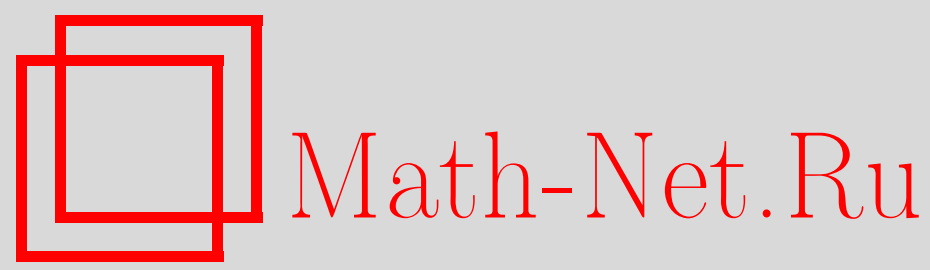

Б. Г. Тасоев, О рациональных приближениях некоторых чисел, Матем. заметки, 2000, том 67, выпуск 6, 931-937

DOI: https://doi.org/10.4213/mzm911

Использование Общероссийского математического портала Math-Net.Ru подразумевает, что вы прочитали и согласны с пользовательским соглашением http://www.mathnet.ru/rus/agreement

Параметры загрузки:

IP : 3.89 .197 .203

26 апреля 2023 г., 15:59:23

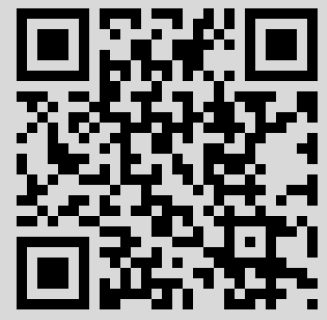


УДК 511.3

\section{О РАЦИОНАЛЬНЫХ ПРИБЛИЖЕНИЯХ НЕКОТОРЫХ ЧИСЕЛ}

\section{Б. Г. Тасоев}

В статье устанавливается точный порядок приближения некоторых чисел рациональньми. Основным инструментом для этого служат разложения в регулярные непрерывные дроби. Доказаны также несколько новых таких разложений.

Библиографиия: 8 названий.

В 1978 году Девис [1] доказал, что при любом $\varepsilon>0$ существует бесконечно много рациональных чисел $p / q$, удовлетворяющих неравенству

$$
\left|e-\frac{p}{q}\right|<\left(\frac{1}{2}+\varepsilon\right) \frac{\ln \ln q}{q^{2} \ln q},
$$

и в то же время неравенство

$$
\left|e-\frac{p}{q}\right|<\left(\frac{1}{2}-\varepsilon\right) \frac{\ln \ln q}{q^{2} \ln q}
$$

имеет лишь конечное число решений. Доказательство этого утверждения использовало полученное еще Эйлером разложение числа $e$ в непрерьвную дробь

$$
e=[2 ; 1,2,1,1,4,1,1,6,1, \ldots]=[2 ; \overline{1,2+2 \lambda, 1}],
$$

а также интегральные представления для числителей и знаменателей подходящих дробей этой непрерывной дроби. Мы применили здесь символ $\overline{1,2+2 \lambda, 1}$ для обозначения бесконечной последовательности чисел, получающейся последовательным соединением блоков $1,2+2 \lambda, 1$ при $\lambda=0,1,2, \ldots$ Подобная сокращенная запись будет использоваться и в дальнейшем.

Непрерывная дробь

$$
b_{0}+\frac{a_{1}}{b_{1}+\frac{a_{2}}{b_{2}+\frac{a_{3}}{b_{3}+\cdots}}}
$$

будет записьваться в виде

$$
b_{0}+\frac{a_{1}}{b_{1}}+\frac{a_{2}}{b_{2}}+\frac{a_{3}}{b_{3}}+\cdots
$$

(см. [2]), а в случае $a_{1}=a_{2}=\cdots=1$ обычно применяется запись $\left[b_{0} ; b_{1}, b_{2}, b_{3}, \ldots\right]$.

Основным результатом статьи является следующее утверждение. 
ТЕОРема 1. Пусть число а определено непрерывной дробъю

$$
\alpha=\left[b_{0} ; b_{1}, \ldots, b_{s}, \overline{c_{1}+\lambda d_{1}, \ldots, c_{m}+\lambda d_{m}}\right]
$$

где все $b_{i}, c_{i}, d_{i} \in \mathbb{Z}$, причем $d_{i}$ неотричательны, а $b_{i}, c_{i}, i \geqslant 1$, положительны. Пусть также $\Omega$ - количество чисел $d_{i}$, отличных от нуля, $\Omega>0, u$

$$
d=\max _{1 \leqslant i \leqslant m} d_{i}
$$

Тогда при с $=\Omega /$ и июбом $\varepsilon>0$ справедливы утверждения:

1) неравенство

$$
\left|\alpha-\frac{p}{q}\right|<(c+\varepsilon) \frac{\ln \ln q}{q^{2} \ln q}
$$

имеет бесконечное число решений в рачиональных числах $p / q$;

2) неравенство

$$
\left|\alpha-\frac{p}{q}\right|<(c-\varepsilon) \frac{\ln \ln q}{q^{2} \ln q}
$$

имеет лишь конечное число решений в рачиональных числах $p / q$.

Если в непрерывной дроби для $\alpha$ все числа $d_{i}=0$, то, как хорошо известно, в этом случае $\alpha$ есть квадратичная иррациональность и не допускает рациональных приближений по порядку лучших, чем $1 / q^{2}$.

Утверждение Девиса следует из теоремы 1 , так как в рассматриваемом случае $\Omega=1$ и $d=2$. Доказательство теоремы 1 использует элементарные свойства непрерывных дробей и существенно проще доказательства теоремы Девиса. Ниже мы приведем ряд других примеров. При этом будут получены новые разложения некоторых чисел в непрерывные дроби указанного типа. Со свойствами непрерывных дробей можно познакомиться по книгам [2], [3].

Следующая лемма относится к произвольным регулярным непрерывным дробям, не обязательно имеющим вид, указанньй в теореме 1.

Лемма 1. Пусть $\alpha=\left[a_{0}, a_{1}, a_{2}, \ldots\right]$ - регулярная непрерывная дробь и $p_{n} / q_{n}$, $n \geqslant 0,-$ последовательность ее подходящих дробей. Тогда справедливы неравенcmsa

$$
\begin{gathered}
\frac{1}{\left(a_{n+1}+2\right) q_{n}^{2}}<\left|\alpha-\frac{p_{n}}{q_{n}}\right|<\frac{1}{a_{n+1} q_{n}^{2}}, \quad n \geqslant 1, \\
a_{1} \cdots a_{n} \leqslant q_{n} \leqslant F_{n} \cdot a_{1} \cdots a_{n}, \quad n \geqslant 1,
\end{gathered}
$$

где $F_{n}-$ последовательность Фибоначчи. 
ДокАЗАТЕЛЬСтво. По существу все неравенства этой леммы хорошо известны. Согласно [3, гл. 24] справедливо равенство

$$
\left|\alpha-\frac{p_{n}}{q_{n}}\right|=\frac{1}{\left(\alpha_{n+1} q_{n}+q_{n-1}\right) q_{n}}
$$

где $\alpha_{n+1}$ есть полное частное непрерывной дроби. Отсюда в силу неравенств $a_{n+1} \leqslant$ $\alpha_{n+1}<a_{n+1}+1$ и $0 \leqslant q_{n-1} \leqslant q_{n}$ следуют оценки $(2)$.

Для доказательства неравенств (3) воспользуемся индукцией. При $n=1$ и $n=2$ неравенства (3), очевидно, выполняются. При $n \geqslant 2$ из равенства $q_{n+1}=a_{n+1} q_{n}+q_{n-1}$ следует, что $a_{n+1} q_{n} \leqslant q_{n+1} \leqslant a_{n+1}\left(q_{n}+q_{n-1}\right)$. Из этих неравенств в силу индуктивного предположения и рекуррентного уравнения $F_{n+1}=F_{n}+F_{n-1}$ для чисел Фибоначчи легко вьводятся неравенства (3).

Мы будем применять доказанную лемму к числу $\alpha$ из теоремы 1 . В этом случае $a_{n}=b_{n}$ при $n \leqslant s$. Если же $n>s$, определим целые числа $t$ и $r$ равенством $n-s=m t+r$, $1 \leqslant r \leqslant m$. Тогда $a_{n}=c_{r}+t d_{r}$.

Лемма 2. В условиях теоремы 1 при $n \rightarrow \infty$ справедлива асимптотическая формула

$$
\ln \left(a_{1} \cdots a_{n}\right)=\frac{\Omega}{m} \cdot n \ln n+O(n) .
$$

ДоКАЗАТЕЛЬСтво. Если в левой части (4) отбросить все сомножители, отвечающие числам $b_{i}$, а также числам $c_{i}+\lambda d_{i}$ c $d_{i}=0$, то произошедшая от этого ошибка будет иметь величину $O(n)$. Поэтому лемму 2 достаточно доказать в предположении, что $s=0$ и при любом $j$ справедливо условие $d_{j} \neq 0$, т.е. $\Omega=m$. Теперь утверждение легко вьводится с помощью равенства

$$
c_{i}\left(c_{i}+d_{i}\right)\left(c_{i}+2 d_{i}\right) \cdot\left(c_{i}+t d_{i}\right) \cdot \Gamma\left(\frac{c_{i}}{d_{i}}\right)=d_{i}^{t+1} \Gamma\left(\frac{c_{i}}{d_{i}}+t+1\right)
$$

и ассимптотической формулы для логарифма гамма-функции.

ДокАЗАТЕЛЬСтво тЕоремы 1. Докажем сначала второе утверждение теоремы 1. Если рациональное число $p / q$ не является подходящей дробью к числу $\alpha$, то справедливо неравенство (см. [3, гл. 25])

$$
\left|\alpha-\frac{p}{q}\right| \geqslant \frac{1}{2 q^{2}}
$$

и, следовательно, неравенство из утверждения 2) теоремы нарушается при больших $q$.

Если $p / q=p_{n} / q_{n}, n \geqslant s$, есть подходящая дробь к числу $\alpha$, то определим целые числа $t$ и $r$ так, чтобы $n+1-s=m t+r, 1 \leqslant r \leqslant m$. Тогда $a_{n+1}=c_{r}+t d_{r}$.

Если $d_{r}=0$, то из левого неравенства (2) следует, что

$$
\left|\alpha-\frac{p_{n}}{q_{n}}\right| \geqslant \frac{1}{\left(c_{r}+2\right) q_{n}^{2}} .
$$

Это противоречит неравенству из утверждения 2) теоремы 1 при больших $n$. 
Если же $d_{r} \neq 0$, то из левого неравенства $(2)$ находим

$$
\left|\alpha-\frac{p_{n}}{q_{n}}\right| \geqslant \frac{1}{\left(t d_{r}+c_{r}+2\right) q_{n}^{2}} .
$$

Из (3) и леммы 2, учитьвая, что $\ln F_{n}=O(n)$, находим

$$
\ln q_{n}=\ln \left(a_{1} \cdots a_{n}\right)+O(n)=\frac{\Omega}{m} \cdot n \ln n+O(n)
$$

и

$$
t=\frac{1}{\Omega} \cdot \frac{\ln q_{n}}{\ln \ln q_{n}} \cdot(1+o(1))
$$

при $t \rightarrow \infty$. Теперь из (5) следует, что

$$
\left|\alpha-\frac{p_{n}}{q_{n}}\right| \geqslant \frac{\Omega}{d_{r}} \cdot \frac{\ln \ln q_{n}}{q_{n}^{2} \ln q_{n}} \cdot(1+o(1)) .
$$

Поскольку $d_{r} \leqslant d$, то (7) противоречит неравенству из утверждения 2$)$ теоремы 1 при достаточно больших $t$.

Итак, любое решение $p / q$ неравенства из утверждения 2) имеет знаменатель, ограниченньй некоторой константой, зависящей от $\alpha$. Этим доказательство утверждения 2) завершается.

Для доказательства утверждения 1) выберем $r$ так, чтобы $d_{r}=d$, и положим $n+1=$ $s+m t+r$ при произвольно выбранном натуральном числе $t$. Тогда $a_{n+1}=c_{r}+t d$ и из правого неравенства (2) находим

$$
\left|\alpha-\frac{p_{n}}{q_{n}}\right|<\frac{1}{\left(t d+c_{r}\right) q_{n}^{2}},
$$

что в силу (6) приводит к неравенству

$$
\left|\alpha-\frac{p_{n}}{q_{n}}\right|<\frac{\Omega}{d} \cdot \frac{\ln \ln q_{n}}{q_{n}^{2} \ln q_{n}} \cdot(1+o(1)),
$$

выполняющемуся при всех натуральных $t$. Это означает, что подходящие дроби $p_{n} / q_{n} \mathrm{c}$ $n+1=s+m t+r$ при $r$, определенном равенством $d_{r}=d$, и любом достаточно большом целом $t$ удовлетворяют неравенству из пункта 1) теоремы 1.

Выведем теперь ряд следствий теоремы 1.

СлЕДСТВИЕ 1. Число

$$
\operatorname{th} \frac{2}{a}=\frac{e^{2 / a}-1}{e^{2 / a}+1}, \quad a \in \mathbb{Z}, \quad a>0,
$$

удовлетворяет утверждению теоремы 1 с константой $c=1 /(2 a)$.

Это утверждение следует из доказанного в 1737 году Эйлером разложения $\operatorname{th}(2 / a)=$ $[0 ; \overline{(1+2 \lambda) a}]$. Отметим, что в работе [4] доказано лишь существование константы $c$, для которой вьполняется второе из утверждений теоремы 1 для числа $\operatorname{th}(1 / a)$. В связи с подобными результатами см. также [5]. 
СлеДСТВИЕ 2. Числа $e^{2}, e^{1 / a} u e^{2 / b}$ при целых $a, b, a \geqslant 2, b \geqslant 3, b$ нечетно, удовлетворяют утверждению теоремы 1 с константами с, равными соответственно $1 / 4,1 /(2 a), 1 /(4 b)$.

Это утверждение вьполняется в силу следующих классических разложений в непрерьвную дробь (см. [6, гл. 14]):

$$
\begin{gathered}
e^{2}=[7 ; \overline{2+3 \lambda, 1,1,3+3 \lambda, 18+12 \lambda}], \quad e^{1 / a}=[1 ; \overline{(1+2 \lambda) a-1,1,1}] \\
e^{2 / b}=\left[1 ; \overline{\frac{b-1}{2}+3 b \lambda, 6 b+12 b \lambda, \frac{5 b-1}{2}+3 b \lambda, 1,1}\right]
\end{gathered}
$$

Отметим, что утверждения относительно чисел $e^{1 / a}, e^{2 / b}$ доказаны в работе Девиca [7].

В следующей теореме мы приведем еще ряд разложений в регулярные непрерывные дроби, из которых при помощи теоремы 1 могут быть получены результаты о рациональных приближениях.

ТЕОРема 2. Справедливы следуюшие разложсения в непрерывные дроби:

$$
\begin{aligned}
& \sqrt{\frac{v}{u}} \operatorname{th} \frac{1}{\sqrt{u v}}=[0 ; \overline{(4 \lambda+1) u,(4 \lambda+3) v}], \quad u, v \in \mathbb{N}, \\
& a e^{1 / a}=[a+1 ; \overline{2 a-1,2 \lambda+2,1}], \quad a \in \mathbb{N}, \\
& a^{-1} e^{1 / a}=[0 ; a-1,2 a, \overline{1,2 \lambda+2,2 a-1}], \quad a \in \mathbb{Z}, \quad a>1, \\
& 2 e=[5 ; 2, \overline{3,2 \lambda+2,3,1,2 \lambda+2}] \text {, } \\
& 3 e=[8 ; 6,2, \overline{5,2 \lambda+2,5,1,2 \lambda+2,5,1,2 \lambda+2,1}] \text {, } \\
& 4 e=[10 ; 1,6,1,7,2, \overline{7, \lambda+2,7,1, \lambda+1,1}] \text {, } \\
& \frac{1}{2} e=[1 ; 2, \overline{2 \lambda+1,3,1,2 \lambda+1,1,3}] \text {, } \\
& \frac{1}{3} e=[0 ; 1,9, \overline{1,1,2 \lambda+1,5,1,2 \lambda+1,1,1,26+18 \lambda}], \\
& \frac{1}{4} e=[0 ; 1,2,8,3, \overline{1,1,1, \lambda+1,7,1, \lambda+1,2}] \text {, } \\
& \operatorname{tg} \frac{1}{a}=[0 ; a-1, \overline{1,(2 \lambda+3) a-2}], \quad a \in \mathbb{Z}, \quad a>1, \\
& \operatorname{tg} 1=[1 ; \overline{2 \lambda+1,1}] \text {, } \\
& \sqrt{a} \operatorname{tg} \frac{1}{\sqrt{a}}=[1 ; \overline{(4 \lambda+3) a-2,1,4 \lambda+3,1}], \quad a \in \mathbb{N}, \\
& \frac{1}{\sqrt{a}} \operatorname{tg} \frac{1}{\sqrt{a}}=[0 ; a-1, \overline{1,4 \lambda+1,1,(4 \lambda+5) a-2}], \quad a \in \mathbb{Z}, \quad a>1, \\
& 2 \operatorname{tg} 1=[3 ; 8, \overline{1,3 \lambda+2,2,3 \lambda+3,12 \lambda+20}], \\
& \frac{1}{2} \operatorname{tg} 1=[0 ; 1,3, \overline{1+3 \lambda, 1,12+2 \lambda, 1,3+3 \lambda, 2}] \text {. }
\end{aligned}
$$

Для доказательства этой теоремы используются классические разложения значений аналитических функций в нерегулярные непрерывные дроби, а также следующее утверждение. 
ЛЕмМА 3. Пусть $r_{0}=1, r_{1}, r_{2}, \ldots$ - ненулевые числа $и$

$$
a_{n}^{*}=r_{n} r_{n-1} a_{n}, \quad n=1,2,3, \ldots, \quad b_{n}^{*}=r_{n} b_{n}, \quad n=0,1,2, \ldots
$$

Тогда непрерывная дробь

$$
b_{0}^{*}+\frac{a_{1}^{*}}{b_{1}^{*}}+\frac{a_{2}^{*}}{b_{2}^{*}}+\cdots
$$

сходится к тому жее числу, что и дробъ (1).

ДокАЗАТЕЛЬСТво. См. [2, теорема 2.6].

ДоКАЗАТЕЛЬСТВо ТЕОРЕМЫ 2. Чтобы доказать равенство (8), положим $x=\sqrt{u v}$ в равенстве $\operatorname{th}(1 / x)=[0 ; \overline{(2 \lambda+1) x}]$ и воспользуемся леммой 3 с $r_{0}=1, r_{2 k+2}=\sqrt{v / u}$, $r_{2 k+1}=\sqrt{u / v}, k \geqslant 0$.

Разложения (9)-(16) доказьваются похожими методами. Для доказательства, например, равенства (9) положим в разложении [2, равенство (6.1.37)] $x=1 / a$ и применим к результату лемму 3 с $r_{0}=1, r_{2 k}=a, r_{2 k-1}=1, k \geqslant 1$. В результате получится разложение

$$
a e^{1 / a}=a+\frac{1}{1}-\frac{1}{2 a}+\frac{1}{3}-\frac{1}{2 a}+\frac{1}{5}-\frac{1}{2 a}+\frac{1}{7}-\cdots
$$

Обозначим через $P_{n} / Q_{n}$ и $p_{n} / q_{n}$ последовательности подходящих дробей для правых частей (23) и (9) соответственно. Индукцией по $n$ легко доказать, что

$$
\frac{P_{2 n+1}}{Q_{2 n+1}}=\frac{p_{3 n}}{q_{3 n}}, \quad \frac{P_{2 n+2}}{Q_{2 n+2}}=\frac{p_{3 n+1}}{q_{3 n+1}}, \quad n \geqslant 0 .
$$

Это доказьвает равенство (9).

Равенства (17)-(22) также доказываются похожими способами. Например, для доказательства равенства (17) воспользуемся разложением

$$
\operatorname{tg} x=\frac{x}{1}-\frac{x^{2}}{3}-\frac{x^{2}}{5}-\frac{x^{2}}{7}-\frac{x^{2}}{9}-\cdots
$$

(см. [2, равенство (6.1.55)]). Четная часть непрерьвной дроби из (18) имеет вид (см. [2, теорема 2.10])

$$
1+\frac{1}{2}-\frac{1}{5}-\frac{1}{7}-\frac{1}{9}-\cdots
$$

Сравнивая эту дробь с (24) при $x=1$, легко получаем, что (24), а вместе с тем и непрерьвная дробь из (18) равны $\operatorname{tg} 1$.

Отметим, что все разложения теоремы 2 в литературе не встречались.

Укажем еще одну теорему, доказываемую так же, как и теорема 1. 
TЕОРемА 3. Пусть $a_{0}, a>1, m>1-$ иелье числа $и$

$$
\alpha=[a_{0} ; \underbrace{\overline{a^{\lambda}, \ldots, a^{\lambda}}}_{m}]_{\lambda=1}^{\infty} .
$$

Тогда при $c=1 / \sqrt{a}$ и любом $\varepsilon>0$ справедливы утвержсения:

1) неравенство

$$
\left|\alpha-\frac{p}{q}\right|<(c+\varepsilon) q^{-2-\sqrt{2 \ln a /(m \ln q)}}
$$

имеет бесконечное число решений в рациональных числах $p / q$;

2) неравенство

$$
\left|\alpha-\frac{p}{q}\right|<(c-\varepsilon) q^{-2-\sqrt{2 \ln a /(m \ln q)}}
$$

имеет лишь конечное число решений в рациональных числах $p / q$.

Пример к теореме 3 дает следующее разложение, доказанное в работе [8]:

$$
\frac{\sum_{s=0}^{\infty} a^{-(s+1)^{2}} \cdot \prod_{m=0}^{s}\left(a^{2 m}-1\right)^{-1}}{\sum_{s=0}^{\infty} a^{-s^{2}} \cdot \prod_{m=0}^{s}\left(a^{2 m}-1\right)^{-1}}=\left[0 ; a, a^{2}, a^{3}, a^{4}, \ldots\right]
$$

\section{СПИСОК ЦИТИРОВАННОЙ ЛИТЕРАТУРЫ}

[1] Davis C.S. Rational approximations to e // J. Austral. Math. Soc. Ser. A. 1978. V. 25. P. 497-502.

[2] Джоунс У., Трон В. Непрерывные дроби. М.: Мир, 1985.

[3] Бухштаб А. А. Теория чисел. М.: Госпедиздат, 1960.

[4] Shiokawa I. Rational approximations to the values of certain hypergeometric functions // Number Theory and Combinatorics (Japan, 1984). Singapore: World Sci. Publ., 1985. P. $353-367$.

[5] Иванков П. Л. О приближении значений некоторых функций // Вестн. МГУ. Сер. 1. Матем., мех. 1994. № 4. С. 12-15.

[6] Perron O. Die Lehre von den Kettenbrüchen. New York: Chelsea, 1929.

[7] Davis C.S. A note on rational approximation // Bull. Austral. Math. Soc. Ser. A. 1979. V. 20. P. 407-410.

[8] Тасоев Б. Г. О некоторых задачах в теории цепных дробей // Тр. Тбилисского ун-та, 1984. C. $53-84$. 